\title{
AUTOMATIC DETECTION OF LANDSLIDES IN OBJECT-BASED ENVIRONMENT USING OPEN SOURCE TOOLS
}

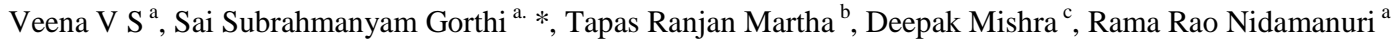 \\ ${ }^{a}$ Dept. of Earth and Space Sciences, Indian Institute of Space Science and Technology, Trivandrum, India \\ ${ }^{\mathrm{b}}$ Geosciences Group, National Remote Sensing Centre, Hyderabad, India \\ ${ }^{c}$ Dept. Of Avionics, Indian Institute of Space Science and Technology, Trivandrum, India \\ email: saisubrahmanyam.gorthi@gmail.com
}

KEY WORDS: Simple Linear Iterative Clustering, Object Based Image Analysis, Green Normalized Difference Vegetation Index, Digital Elevation Model, Principal Component Analysis, Speeded Up Robust Features

\begin{abstract}
:
Automatic detection of landslides from very high resolution satellite images is a prerequisite for rapid damage assessment and supporting disaster management activities. In this study, a novel method using open source tools was developed for extracting landslides from bi-temporal satellite images based on Object Based Image Analysis (OBIA). The methodology employed involves image segmentation followed by elimination of non-landslide candidates using object based change detection techniques and lastly, unsupervised classification. Brightness of a post-landslide image is higher in comparison to its pre-landslide image hence a suitable threshold value for post image brightness was set to demarcate the landslide affected regions from the other land cover types. Further, landslide diagnostic parameters such as difference in Green Normalized Difference Vegetation Index (GNDVI), Digital Elevation Model (DEM, slope, Principal Component Analysis (PCA) and difference in Top of Atmosphere (ToA) values were used to eliminate challenging false candidates such as snow cover, barren land and river sediments. The objects retained after eliminating false candidates are then classified into two classes using k-means clustering algorithm. The local features associated with an image can be computed by finding the key points using a Speeded Up Robust Feature (SURF). Performance of this method was investigated using Resourcesat-2 LISS-IV multispectral $(5 \mathrm{~m})$ bi-temporal satellite image covering parts of Uttarakhand state in India. Results show that the proposed methodology will aid rapid inventorisation of landslides.
\end{abstract}

\footnotetext{
* Corresponding author
} 


\section{INTRODUCTION}

Landslide inventory from remote sensing images is essential. Any effort to reduce the risk of landslide should start with an inventory, a detailed map showing where the landslides have occurred. The possibility of detection of historical landslides provides a reference strategy for setting long term disaster management activities while rapid inventorisation of newly triggered landslide is less for immediate disaster response. Therefore, an automatic technique to detect landslides from very high resolution satellite imaging is crucial in overcoming the limitations that exist in traditional field inspection method and for the efficient utilization of high resolution data availability.

Generally, the regions corresponding to a landslide region are identified based on its spectral characteristics. Detection of landslide with the traditional pixel based approach seems less relevant because the same pixel intensity value may indicate different land cover class like barren land, cloud cover, etc. So it becomes too difficult for finding a criterion for efficient land cover classification as well. In this research work, we develop an OBIA method to automatically detect landslides from multispectral remote sensing imaging which treats image into various image regions rather than traditional pixel based approach. It tries to overcome the limitation of traditional pixel based approach and provide segmentation based object oriented analysis to improve the classification accuracy.

Remote sensing techniques have been widely used from 1990 for landslide research and for this purpose different spatial and spectral resolution images have been employed. Research work conducted so far includes photo-interpretation and inventory of large landslides, determinant factor analysis, stereo-plotting of movements, and automatic detection by textural analysis. The irregular boundaries and surface textures of landslides mean that they often produce characteristic features that can be enhanced in remote sensing imaging through textural analysis. Over the past few decades, many studies have been carried for landslide detection. Interpretations of landslides from aerial photographs have been developed from on-screen images. Li-Wei et.al (2006) used FORMOSAT 2 images for identification of landslides because of its advantage of low cost and frequent revisit. Their technique explores discriminative features, such as texture and topological features. Texture features were extracted using log polar wavelet packet transformation whereas for topological feature slope is derived. Finally a contextual based classifier that combines spectral and spatial information was used for homogeneous object identification. Zhongbin Li et.al (2016) developed a semi-automated approach from bi-temporal satellite images which uses a change detection based threshold analysis to generate initial zero level curve. Method includes generating difference image using change vector analysis, detecting landslide candidates using change detection based threshold method and removing false candidates using some morphological operations. Landslide boundaries are detected using edge based and region based level set evolution. HaiRong Ma et.alb (2016) proposed a method to detect shallow landslides using Worldview2 images. Features like high soil brightness and low vegetation coverage helped them to identify shallow landslides on remote sensing images. To eliminate back scrap, shadow index was used. FORMOSAT-2 multispectral imagery provides poor landslide identification since it considers only spectral features. LiDAR data have provided landslide maps in forested areas. In general, the research work conducted for Landslide Inventory Map methods can be classified into three groups: Pixel based approaches, Object based approaches and hybrid approaches. With respect to change detection algorithm, landslides are land cover changes and can be detected by using pre and post landslide images. Usually change detection method based on pixel approach has the limitation of 'salt and pepper effect'. There comes the significance of grouping homogeneous pixels into an object or into 'super pixels'.

In this study, an algorithm to automatically detect landslide from satellite imaging was developed by incorporating a change detection based threshold analysis and followed by unsupervised classification. For change detection analysis, we considered bi-temporal satellite images. Methodology was developed using open source tools where some contextual features were used at object level to detect landslides. Segmentation based object oriented analysis with certain optimal parameters were used to segment image into homogeneous image objects. Thus segments become the object primitives and they can be used further for image analysis. Simple Linear Iterative Clustering algorithm was used to cluster image objects. It was followed by a change detection technique, which enhanced the change detected regions by comparing pre and post landslide images. One of the most important properties observed in post landslide image is the increase in the brightness level for the areas surrounded by the landslide affected region. Parameters that can be considered for discriminating landslides from change detected regions were considered at object level. They were used with suitable threshold values based on our domain knowledge. From the change detected output, key points were computed. Associated with each key point, key point descriptors were described manually. Further on this array, K-means clustering algorithm was performed to cluster each objects into their respective classes.

\section{METHODOLOGY}

Automatic detection of landslides from remote sensing imagery consists of the following sub-blocks:

1) Image segmentation using Simple Linear Iterative Clustering algorithm (SLIC).

2) Elimination of non-landslide candidates using object based change detection technique.

3) Classification for discriminating landslides.

\subsection{Bi-temporal Satellite Images}

Test input images used for this research work was a Resourcesat-2 LISS-IV multispectral (5m) bi-temporal satellite image covering parts of Uttarakhand state in India. Bi-temporal satellite images are the images captured at two different instant of time of the same place and will be considered as Post and Pre-landslide images throughout this paper.

\subsection{Segmentation of Post Landslide Image Using SLIC}

A necessary prerequisite for Object Oriented Image Analysis is successful image segmentation. To obtain useful information from an image, the segmentation process splits an image into unclassified "object primitives" that forms the basis for the image objects and the rest of the image analysis. Segmentations, and the resulting characteristics of object primitives and eventual image objects, are based on shape, size, color, and pixel topology controlled through parameters set by the user. The values of the parameters define how much influence spectral and spatial characteristics of the image layers will have in defining the shape and size of the image objects. 


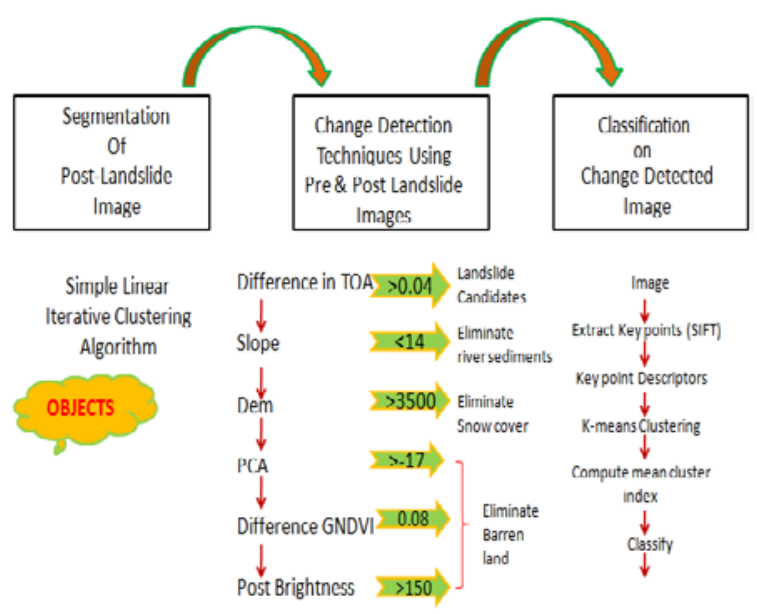

Figure 1. Block diagram of the proposed scheme

The user modifies the settings depending on the objective, as well as image quality, bands available, and image resolution. As a general rule, 'good' image objects should be as large as possible, but small enough to show contours of interest and to serve as building blocks for objects of interest not yet identified. In this research work, we have used SLIC to create image objects which decompose an image into visually homogeneous region based on the spatially localized version of K-means clustering algorithm. It has two parameters like region size and regularizer. The following pseudo code was used for computing SLIC.

1. Image is divided into grid with region size. 2. Centre of each grid is used to initialize corresponding Kmeans.

3. Finally, K mean centers and clusters are refined by Lloyd algorithm. Lloyd's algorithm starts by an initial placement of some number $\mathrm{k}$ of point sites in the input domain. In mesh smoothing applications, these would be the vertices of the mesh to be smoothed; in other applications they may be placed at random, or by intersecting a uniform triangular mesh of the appropriate size with the input domain. It then repeatedly executes the following relaxation step: -The Voronoi diagram of the $\mathrm{k}$ sites is computed. -Each cell of the Voronoi diagram is integrated and the centroid is computed. •Each site is then moved to the centroid of its Voronoi cell. Voronoi diagram construction algorithms can be highly nontrivial, especially for inputs of dimension higher than two, the steps of calculating this diagram and finding the centroids of its cells may be approximated by a suitable discretization in which, for each cell of a fine grid, the closest site is determined, after which the centroid for a site's cell is approximated by averaging the centers of the grid cells assigned to it. During K-mean iteration each pixel can be assigned to only $2 \times 2$ centers corresponding to grid tiles adjacent to pixel. Regularizer, $\lambda$ sets the tradeoff between clustering appearance and spatial regularization.

$$
\lambda=\frac{\text { Regularizer }}{\text { Region Size }}
$$

SLIC starts by dividing the image domain into regular grid $\mathrm{MxN}$ tile where

$$
M=\frac{\text { Image Width }}{\text { Region Size }} ; \quad N=\frac{\text { Image height }}{\text { Region Size }}
$$

Region is initialized with each grid center

$$
\mathbf{x}_{\mathbf{i}}=\operatorname{round}[\mathrm{M}] ; \quad \mathbf{y}_{\mathbf{i}}=\operatorname{round}[\mathrm{N}]
$$

To avoid placing these centers on the top of discontinuities (edges) centers are moved in a 3x3 neighborhood to minimize edge strength

$$
\text { edge }(\mathrm{x}, \mathrm{y})=\left\|I_{(X+1, Y)}-I_{(X-1, Y)}\right\|^{2}+\| I_{(X, Y+1)}-I_{(X, Y-1)}
$$

Regions are obtained by running K-means clustering algorithm started from centers. K means uses a Lloyd algorithm, i.e., to reestimating centers as the average of corresponding feature vectors of the pixel assigned to them. After convergence SLIC eliminates any connected region whose area is less than min [Region Size] pixels. This is done by merging region to neighbours.

\subsection{Proposed Change Detection rule set}

Usually for landslide regions the brightness is more as compared to its corresponding pre-landslide region, a change detection method was proposed to effectively detect change detected regions (Martha et al., 2012). For change detection algorithm, we considered some suitable parameters like difference in Top of Atmosphere (ToA) reflectance among pre and post landslide images, difference in Green Normalised Difference Vegetation Index (GNDVI) among pre and post landslide images, fourth and fifth principal component, slope, DEM to effectively eliminate false candidates from the change detected regions. Here, we explore two methods for change detection: 1) Feature based thresholding, and 2) Change detection based thresholding. The following sub-sections descried these methods in detail.

\subsubsection{Feature Based Threshloding}

In this subroutine, we set appropriate threshold for each feature value to eliminate the non-landslide candidates from the Post image. The thresholds used are discussed below.

2.3.1.1. Difference in ToA: Based on our domain knowledge, we can say that the brightness value for a landslide occurred region is more as compared to its corresponding pre-landslide region. This is due to the exposure of fresh rock surface. So a difference in ToA among pre and post landslide image were considered to effectively detect the change occurred regions. We compute threshold conditions for detecting those regions. There are three ToA bands (NIR, green and red), and the difference of their means gives the difference in ToA of the two images. The equation used for the calculation of difference in brightness is,

$$
\begin{aligned}
\text { Difference in ToA }= & \text { Post image } \\
& \text { image }_{(\text {ToA band1 }+ \text { ToAband } 1+\text { ToAband2 }+ \text { ToAband } 3)}
\end{aligned}
$$

2.3.1.2. Difference in GNDVI: GNDVI uses NIR (Near InfraRed) and green bands of the electromagnetic spectrum. It is same as to the NDVI, but here red band is replaced by green band. Equation for the calculation of GNDVI is,

$$
\frac{\text { TOA }_{\text {NIR }}-\text { TOA }}{\text { Green }}
$$

Significance for choosing GNDVI parameter for landslide detection:

$$
\text { - } \quad \text { It can be used for eliminating barren lands. }
$$


- GNDVI is very sensitive to landslides; decrease in GNDVI value in post image indicates vegetation loss due to landslides.

- It can be used for detecting small scale changes.

2.3.1.3. Principal component analysis: PCA is an image analysis technique used for reducing the dimensionality of a data set. Some of the original bands may be highly correlated and, to save on data storage space and computing time, such bands should be combined into new less correlated eigen images by PCA. Newly triggered landslides were primarily concentrated in the PC4. Because of high brightness, some barren land may be misclassified as landslide candidates. To effectively eliminate such false candidates PCA of stacked pre and post landslide images were used for separation of barren land. Threshold value for removal of barren land is set to -17 .

2.3.1.4. DEM: DEM is always used as a generic term, only representing height information. DEM has been widely used in assessment of landslide susceptibility risk. Some challenging features like snow cover, cloud cover have high brightness values which may be misclassified as landslide candidates. So DEM layer is used to eliminate snow covered areas since snow is confined to high altitude regions. Threshold value to eliminate snow cover was taken to be 3500 , in our work. Objects with value greater than or equal to 3500 are considered as snow cover line.

2.3.1.5. Slope: Slope layer is very useful for the identification of the false positives like river and river sand. River sand has high brightness, small slope and low relief. Most of the landslides occur in very steep slope. So it is easy to separate landslides from false positives using slope angle. At some regions river sediments show same brightness characteristics as that of a landslide region which leads to the misclassification of river sediments into landslide candidates. So slope can be used as the feature to identify landslide candidates since landslide occurs in steep slopes. Objects with slope values less than or equal to 14 is considered as river sediments.

2.3.1.6. Post image brightness: Finally post image brightness is used for removal of barren land from landslide candidates. Post image brightness with values less than or equal to 100 is used for discriminating barren land from remaining candidates.

\subsubsection{Change Detection Based Threshold (CDT)}

It can be used for detecting changes among two registered images acquired from same geographical position at different instant of time. CDT comprises of mainly two steps: 1) Difference Image 2) Threshold. In this study pre and post landslide image is considered for the difference image computation. The magnitude of change vector for a specific pixel is defined as

$$
\rho(X)=\sqrt{\sum_{b=1}^{n}\left(X_{b, t_{1}}-X_{b, t_{2}}\right)^{2}}
$$

where $X_{b, t_{1}}$ and $X_{b, t_{2}}$ are pixel values obtained at time $t_{1}$ and $t_{2}$ respectively where ' $b$ ' is the band and ' $n$ ' is the total number of bands. Greater values of $\rho(X)$ indicates that the pixel $X$ have big change from $t_{1}$ to $t_{2}$. Therefore brighter pixels in $\rho(\mathrm{X})$ indicate landslide candidates. False candidates (non landslides) might be present within the difference image. To overcome this, a threshold is set at object level that distinguishes landslide candidates from non landslide ones within the Difference Image (DI). The magnitude of each pixel was compared at object level with the set threshold value. If it is greater than or equal to a threshold, the object is labelled as landslide candidate; otherwise not. The threshold value is formulated as:

$$
\mathrm{X}_{\mathrm{DI}}\left\{\begin{array}{l}
\text { Landslide, } \rho(\mathrm{X}) \geq \bar{\rho}+\alpha^{*} \sigma \\
\text { Nonlandslide, otherwise }
\end{array}\right.
$$

where $X_{D I}$ value of object $X$ in DI. $\bar{\rho}$ and $\sigma$ are mean and standard deviation of $\rho$ respectively. $\alpha$ is fixed as 1.5 .

\subsection{Classification}

After change detection, the objects that left within the image are used for classification. The method involves feature point detection, feature descriptors, visual word dictionary and a classifier (Cheng et.al, 2013). In feature point detection, the pixels which correspond to edges and corners highlights significant information compared to pixels of homogeneous region. Significant pixels can be computed using different approach like SIFT and SURF. They are usually called as key points which indicate local variation of image intensity in the spatial domain. Associated with each key points, descriptors are computed which might be usually SIFT descriptors. But in this work, we have defined the descriptors; the parameters like slope, DEM, PC4 and mean intensity. We observed that these features are apt for landslide identification. Those parameters were considered at object level. i.e., associated with each key point, those parameters values are calculated at object level for the non-zero segments obtained after change detection. K means clustering algorithm was used to cluster key point descriptors in their feature space into two major clusters. Using mean intensity values, DEM, Slope and PC4 the key point descriptors are clustered and encode each key point descriptors by the index of the cluster into landslide class or non-landslide class (Figure 2).

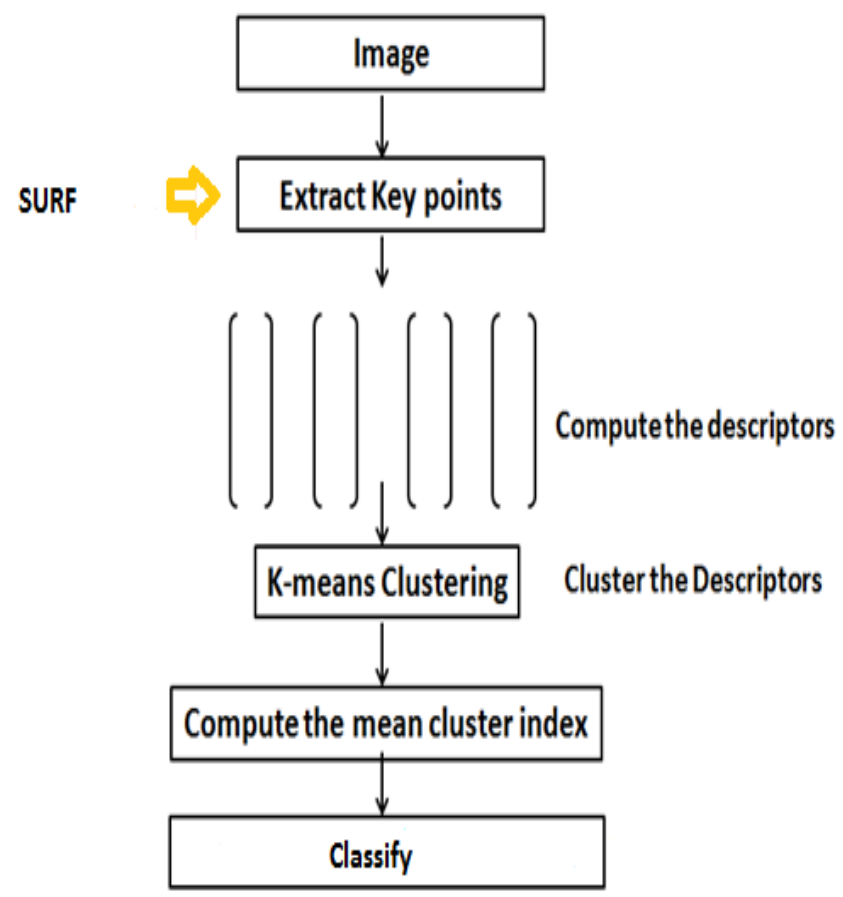

Figure 2. Block diagram for classification scheme 


\section{RESULTS \& DISCUSSION}

For the experimental analysis for landslide detection, bitemporal LISS-IV multispectral images of Chamoli district in Uttarakhand region, which were collected from NRSC, Hyderabad, India, have been used. The ground truth data says that there are 377 landslides in total which includes small, medium and large sizes. In this section, we will discuss our results and inferences drawn out of this exercise.

\subsection{Segmentation}

SLIC segments the post landslide image into objects of different shape and size based on suitable region size. For the current analysis, scale parameter of 30 was used to create objects.

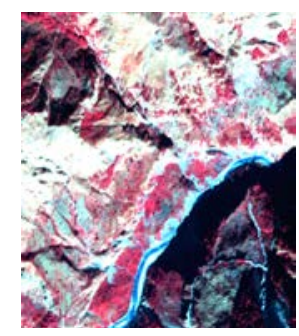

(a) Pre-Segmentation

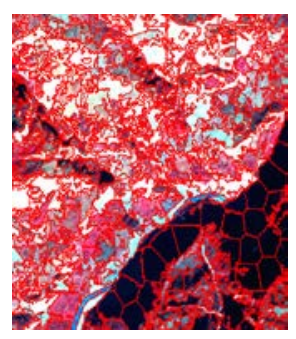

(b) Post-Segmentation
Figure 3. SLIC Segmentation

\subsection{Change Detection}

Two methods of change detection approach were experimented. One was based on proposed change detection rule set (Feature Based Thresholding, Section 2.3.1). Second was based on the difference image concept (Change Detection Based Threshold, Section 2.3.2).

\subsubsection{Results of Proposed Change Detection rule sets}

3.2.1.1. Elimination of false positives based on difference in TOA: The rule set begins with finding those change detected regions by comparing TOA values of pre as well as post images. Landslide objects within the post image have more brightness as compared to corresponding objects in the pre image. Thus, if the threshold value for difference in TOA brightness among the objects was greater than 0.04 , then the object will be considered as landslide candidate. Figure (4) depicts the cropped region within the post landslide image and the black portion indicates the eliminated segment since it doesn't have changes while comparing at object level basis. After taking the difference in TOA for extracting change detected candidates, further elimination of false positives are necessary. To discriminate each one of them and eliminate those false positives, additional parameters and its corresponding threshold conditions were used in this study.

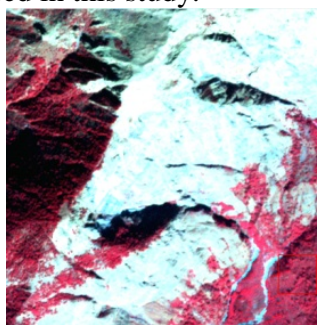

(a) Before ToA thresholding

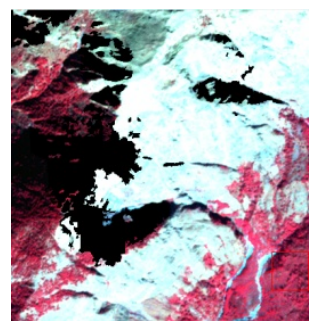

(b) After ToA thresholding
Figure 4. Result after diff. TOA thresholding at object level
3.2.1.2. Elimination of false positives based on slope: It was observed that there exist some river sediments that might have almost the same brightness as that of landslide candidates. Elimination of such regions can be performed well using slope parameter. It was observed that if the mean value of slope within an object is less than or equal to 14 , it indicates river sediments because most of the landslide happens on steep slopes. From the figure (2), it is evident that the river sediments are well eliminated.

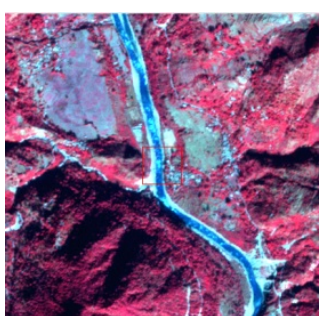

(a) Before Slope thresholding

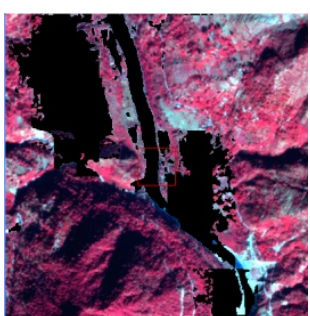

(b) After Slope thresholding
Figure 5.Result after threshold slope at object level

3.2.1.3. Elimination of false positives based on DEM: Once TOA have been calculated, presence of some brightness features like snow, cloud cover may be misclassified as landslides. DEM layer can be used for the elimination of snow covered areas since snow is confined to high altitude regions. For DEM, average value among the object after the segmentation is greater than $3500 \mathrm{~m}$, then the object is confined to be in high altitude and will be considered as a snow cover line. Figure (6) indicates the elimination of snow cover.

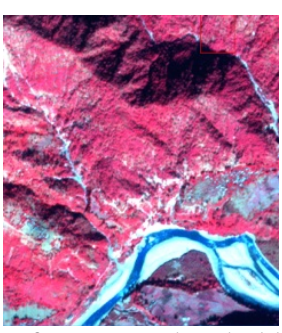

(a) Before DEM thresholding

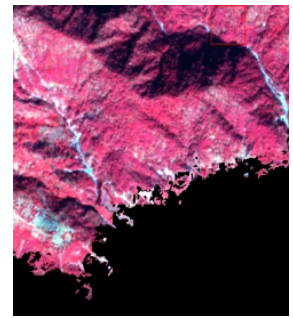

(b) After DEM thresholding
Figure 6. Result after threshold DEM at object level

\subsubsection{Elimination of false positives based on PC4}

Principal Component layers can be used for the elimination of barren land, as is shown in many studies. PC4 and PC5 were used for landslide detection since both are good for detecting landslide triggered regions. Due to the high brightness some barren land may be misclassified as landslide candidates. Computation of PCA was performed by stacking pre and post image layer. Layer stacking was performed on both pre and post landslide images. Many research works suggest that PC4 and PC5 are good features for identifying new landslides. If the average value among the objects within the $4^{\text {th }}$ or $5^{\text {th }}$ principal component was greater than -17 it will be eliminated, hence eliminating the barren land candidates.

\subsubsection{Elimination of false positives based on GNDVI}

GNDVI is a good indicator of landslides because it can be used for the detection of even small scale changes. If the average value of GNDVI among the objects is less than 0.08 , then that condition would eliminate barren lands. Figure (8) indicates 
eliminated barren land by considering difference in GNDVI among the post as well as pre landslide image.

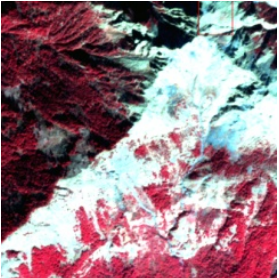

(a) Before PC4 thresholding

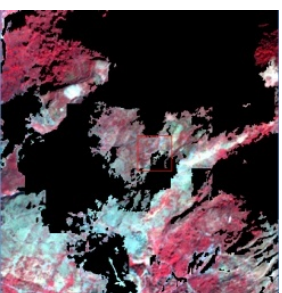

(b) After PC4 thresholding
Figure 7.Result after thresholding PC4
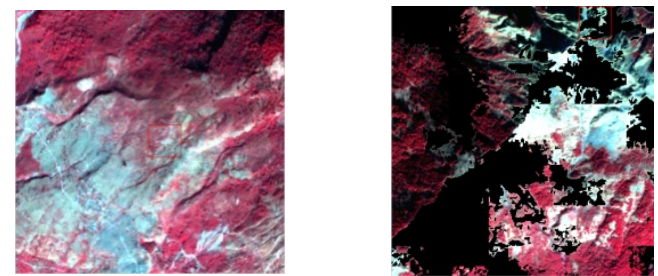

(a) Before GNDVI thresholding (b) After GNDVI thresholding

Figure 8.Result after thresholding diff in GNDVI

3.2.1.6. Elimination of false positives based on post image brightness: Majority of the challenging features is eliminated to a certain extent; but some false positives still exist. Among them barren land can also be eliminated by taking the mean intensity values. Within an image object, if the mean intensity value is less than 110 it indicates that it is barren land, and thus can be eliminated by thresholding.. But it was evident that there still exist some significant regions that need to be further eliminated. Further elimination was done through keypoint detection followed by unsupervised classification, as is discussed in Section 3.3.

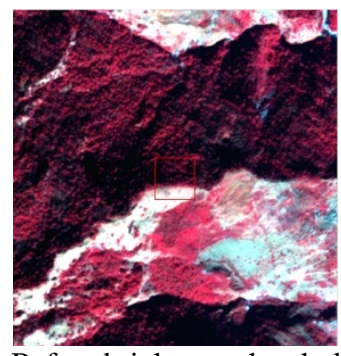

(a) Before brightness threshold

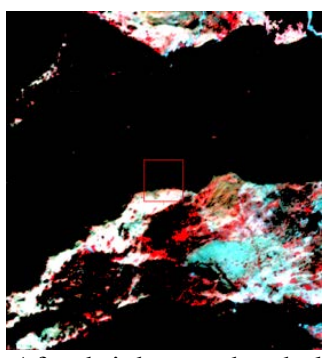

(b) After brightness threshold
Figure 9.Result after threshold brightness values.

\subsubsection{Object level Change Detection based threshold}

Here, a pixel based image analysis technique, which has been converted to object based analysis after segmentation, has been discussed (Hai-Rong, 2016).

3.2.2.1. Elimination of false positives based on difference image: Figure 10 shows the result of difference image threshold at object level obtained by considering 3 bands of pre and post images. Selection of tuning parameter was based on our domain knowledge. From the difference image obtained parameters like mean and standard deviation were computed. Tuning parameter with various trial and error approaches it was opted as 1.5.

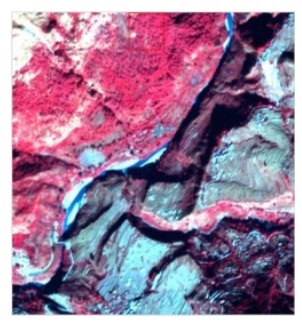

(a) Before CD thresholding

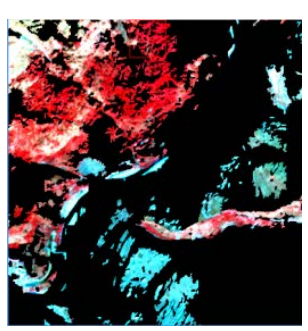

(b) After CD thresholding
Figure 10. Result after CD thresholding at object level

\subsection{Classification}

Change detection was followed by classification. In change detection algorithm, we have considered different parameters for detecting changes, such as detected region which includes the landslide candidates and some false positives as well. Image which obtained after the change detection still includes a large number of false positives. Unsupervised classification using kmeans tries to classify the image into two classes based on the descriptors associated with each of the key points. From the change detected output, the algorithm tries to find the key points by means of SURF algorithm. Figure (11) shows the key points computed over the entire image using SURF detector (Vetrivel, 2016). From the image of dimension 5620 X 4894, about 30,832 corners were detected by applying the threshold value of 10,000 .
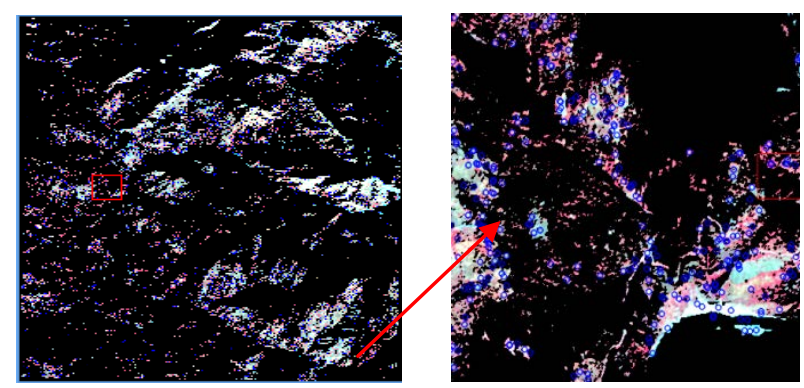

Figure 11. SURF feature detectors

Advantages of using SURF as feature detector is that, key points can be computed over the entire image within a short duration of time. Associated with each key point, key point descriptors are described. In this experiment the descriptors taken are mean intensity value, slope, DEM, PC4 associated with each key point and the mean over its corresponding object. Here, an array was created for all those non-zero objects having key points followed by unsupervised classification (k-means). Two classes were declared and each object was encoded into either of the two class based on their feature vectors.

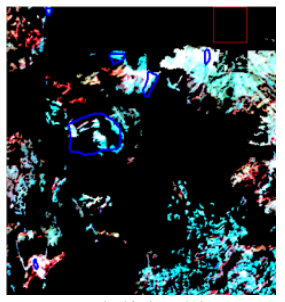

(a) Landslide Class

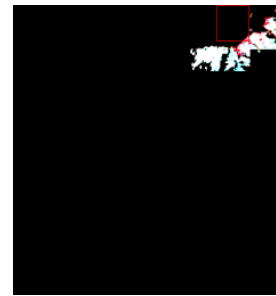

(b) Non Landslide Class
Figure 12. Result obtained after Classification (Blue polygons indicate the overlaid shape files) 


\section{Evaluation Metrics}

In object detection techniques, three measures for evaluating the accuracy of methods are precision, recall and $F$ measures. Precision tells about how accurately the detected objects overlap with the ground truth. Using precision we can measure Fmeasure. Precision measures the fraction of detections that are true positives and Recall measures fraction of positives that are correctly identified. Let as assume Tp, Fp and Fn are the true positive, false positive and false negatives respectively.

Recall

$$
\mathrm{R}=\frac{T_{P}}{T_{P}+F_{n}}
$$

The below tables summarizes the effect of evaluation metric with different key point descriptor and with different non zero segments descriptor followed with parameters like slope, DEM and principal component factors. Table compares the final results performance assessment.

\begin{tabular}{|c|c|c|}
\hline Evaluation Metric & Change Detection & CDT \\
\hline $\mathrm{T}_{\mathrm{p}}$ & 302 & 287 \\
\hline $\mathrm{F}_{\mathrm{n}}$ & 75 & 90 \\
\hline $\mathrm{R}$ & 0.801 & 0.761 \\
\hline
\end{tabular}

Table 1: Summary of the proposed change detection rule set and CVA followed by key point descriptors.

\begin{tabular}{|c|c|c|}
\hline Evaluation Metric & Change Detection & CDT \\
\hline $\mathrm{T}_{\mathrm{p}}$ & 227 & 271 \\
\hline $\mathrm{F}_{\mathrm{n}}$ & 150 & 106 \\
\hline $\mathrm{R}$ & 0.602 & 0.719 \\
\hline
\end{tabular}

Table 2: Summary of the proposed change detection rule set and CVA followed by classification without key point descriptors.

\section{CONCLUSION}

OBIA based extraction of landslides from very high resolution satellite imagery is a promising technology nowadays. This is because of the importance of landslide inventory map in hazard mapping of an area. In this scheme, we have discussed an open source technique to automatically detect landslides of small, medium and of larger types. Based on proposed rule set for change detection and combining the concept of machine learning algorithm false positive elimination within the image have performed to a certain extent. Also, we experimented with a change detection based threshold analysis for false positive elimination based on difference image at object level. But a generalized way to optimize the algorithm still exist which can be further improved at descriptors level. Aforementioned method utilized LISS IV bi-temporal multispectral satellite images of Uttarakhand region. Algorithm shows that for the proposed change detection rule set followed by key point descriptors about $80.1 \%$ recall was obtained. Precision was not calculated due to a large number of false positives in the landslide image. Methodology implies that there exist few more challenging candidates that need to be further eliminated. In future research, we need to solve for such problem by including additional parameters like some texture parameters for enhancing landslide candidates from the current algorithm result.

\section{ACKNOWLEDGEMENTS}

Authors would like to thank Runjhun Chandra, B.Tech (Physical Science) student, IIST, for helping in editing the paper to its final form as per suggestions.

\section{REFERENCES}

[1] Anand Vetrivel, Markus Gerke, Norman Kerle, George vosselman, Identification of structurally damaged areas in airborne oblique images using a Visual-Bag-of-Words approach, Remote sensing 2016

[2] David G Lowe, Distinctive image features from Scale invariant key points, International Journal of Computer Vision, January 2004

[3] Gong Cheng, Junwei Han "A survey on object detection in optical remote sensing images”, ISRPS Journal of Photogrammetry and remote sensing, March 2016, pg.11-28

[4] Gong Cheng, Lei Guo, Tianyun Zhao, Junwei Han, Huihui Li \& Jun Fang "Automatic landslide detection from remote sensing imagery using a scene classification method based on BoVM and Plsa “, International Journal of Remote Sensing, Vol.34,No.1, 10 January 2013,45-59

[5] Hai-Rong Ma, Xinwen Cheng, Lianjn Chen, Haitao Zhang, Hongwei Xiong, Automatic identification of shallow landslides based on Worldview 2 remote sensing images, Journal of applied remote sensing, March 2016, Vol.10, issue 1 [6] Li-Wei Chang,Pi-Feui Hsieh and Ching Weei Lin "Landslide identification based on FORMOSAT-2 multispectral imagery by wavelet based Texture Feature Extraction", IEEE International conference on Geoscience and Remote Sensing Symposium, 2006

[7] Paul L.Rosin, Javier Hervas, Jose I Berredo , Remote sensing image thresholding for landslide motion detection [8] Tapas R. Martha, Norman Kerle, Cees J van Western, Victor Jetten, K. Vinod kumar, Object oriented analysis of mutitemporal panchromatic images for creation of historical landslide inventories, ISRPS Journal of Photogrammetry and remote sensing, January 2012, Vol. 66-issue 1, pg no:105-119 [9] V. Keerthi and A. Senthil Kumar "At-sensor Solar Exoatmospheric Irradiance, Rayleigh Optical Thickness and Spectral parameters of RS-2 Sensors"

[10] Zhongbin Li, Wenzhong Shi, Soe W Myint, Ping Lu, Qunming Wang, Semi automated landslide inventory mapping from bitemporal aerial photographs using change detection and level set method, Remote sensing environment, 2016, pg.215230 